\title{
CROSS TALK IN 1872 RETICON DIODE ARRAYS
}

\author{
G. A. H. WALKER, * R. JOHNSON, AND D. RICHARDSON
}

Department of Geophysics and Astronomy, University of British Columbia, Vancouver, BC V6T 1W5, Canada

B. CAMPBELL, * A. W. IRWIN, AND S. YANG*

Department of Physics and Astronomy, University of Victoria, P.O. Box 1700, Victoria, BC V8W 2Y2, Canada

Received 1990 August 23

\begin{abstract}
If preamplifiers which integrate charge on the video lines are used with the Reticon 1872 they introduce an "odd-even" diode cross talk at the $\sim 1 \%$ level in sharp spectral features because of capacitance between the first and second, and third and fourth, video lines. The effect is stable and can be eliminated with a simple algorithm.
\end{abstract}

Key words: detectors-Reticons-diode arrays-cross talk

\section{Introduction}

The virtues of self-scanning, linear arrays of silicon diodes for highly precise spectrophotometry have been widely demonstrated (e.g., de Strobel and Spite 1987). One of the most successful devices has been the RL 1872 F/30 manufactured by EG\&G Reticon. It can be calibrated photometrically to better than $0.1 \%$ and register single spectra with a signal-to-noise ratio of greater than 1000 per diode (e.g., Walker (1987) or Walker, Johnson, and Yang (1985)). These features have been central to the success of our precision radial-velocity program (Campbell, Walker, and Yang 1988) in which we measure stellarabsorption-line displacements to an accuracy of 0.04 diode spacing. To reach this precision we must also correct our spectra for the effect of an odd-even diode cross talk.

\section{The Cross Talk}

The effect of the cross talk on spectra is best illustrated with an emission line or a strong feature in absorption. Figure 1 (a) shows the $\lambda 8717, R=2$ absorption line from the 3-0 vibration band of hydrogen fluoride in a spectrum taken with the 1872 Reticon and coudé spectrograph of the Canada-France-Hawaii Telescope (CFHT) at a dispersion of $0.07 \AA$ per diode. The absorption line was imposed on the continuous spectrum of an incandescent lamp by passing the light through a cell filled with HF.

The spectrum in Figure 1(a) has been rectified to a continuum and is plotted as a function of diode number. The data were corrected for baseline, differences in diode-to-diode sensitivity, and video-line gain as de-

*Visiting Astronomer, Canada-France-Hawaii Telescope, operated by the National Research Council of Canada, the Centre National de la Recherche Scientifique of France, and the University of Hawaii. scribed by Walker et al. (1985). The signal-to-noise ratio in the continuum is $\sim 3000$. Figure $1(b)$ shows the residuals after fitting a Lorentzian and three Gaussian profiles to the line. The odd-even effect is quite clear. It reaches a maximum amplitude at the steepest part of the profile and changes phase on passing through the line center.

In simple terms, where the intensity is increasing with diode number the signal on each odd-numbered diode is decreased by a fraction of the signal on the subsequent even-numbered diode, and the signal on the even-numbered diode is increased by a fraction of the signal on the subsequent odd-numbered diode and vice versa when the intensity decreases with diode number. The effect is almost certainly caused by the video-line geometry in the device. There are four video lines and, in a given quartet of diodes, each diode is read by a different video line. The video lines emerge from the device in pairs with some capacitative coupling between lines 1 and 2 and between 3 and 4 . With the preamplifier design used in our systems (Walker et al. 1985), in which charge is integrated directly on the video line, such coupling leads to cross talk.

\section{The Algorithm}

One of us (B.C.) has written an algorithm which we use routinely to correct for the odd-even cross talk. As cross talk is only important in the presence of marked spectral gradients, the algorithm operates differentially:

$$
\begin{aligned}
s_{1} & =0.00\left(I_{(i+3)}-I_{(i+1)}\right) \\
I^{\prime}{ }_{(i+2)} & =I_{(i+2)}-\alpha s_{1} \\
s_{2} & =0.00\left(I_{(i+2)}-I_{(i)}\right) \\
I^{\prime}{ }_{(i+1)} & =I_{(i+1)}+\alpha s_{2},
\end{aligned}
$$

where $I(p)$ ( $p: 1$ to 1872$)$ is the spectral intensity, and $\alpha(=0.042)$ is the empirically determined odd-even scale factor. 


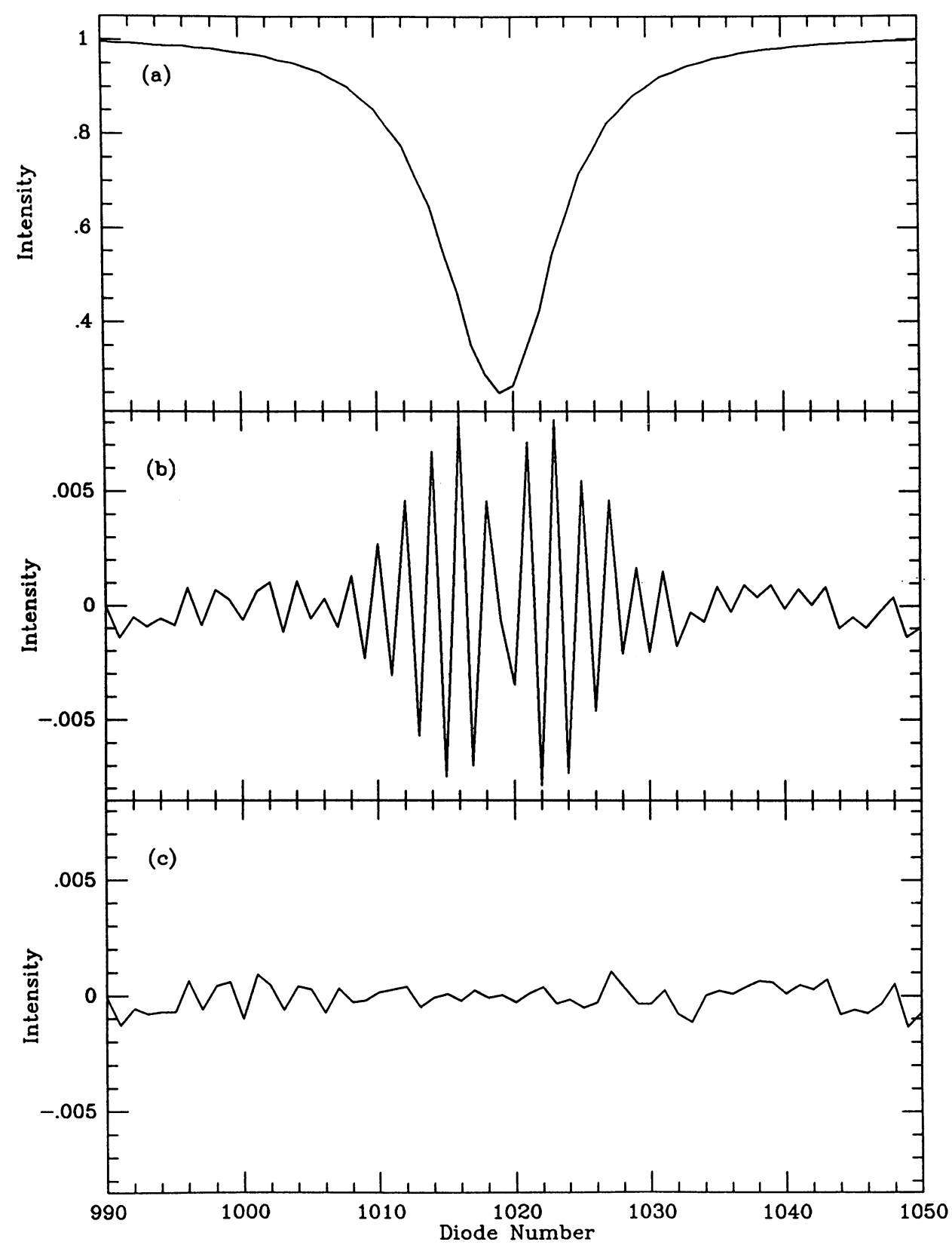

FIG. 1-(a) Profile of the HF $\lambda 8717, R=2(3-0)$ absorption line as a function of diode number after correction for baseline, diode-to-diode sensitivity, and video-line gain - taken with the CFHT 1872 Reticon and coude spectrograph. (b) Residuals of the profile in (a) from a best-fitting theoretical fit of one Lorentzian and three Gaussians. (c) Same as (b) but after correction of the data with the odd-even algorithm.

The effectiveness can be seen in Figure 1(c) which shows residuals from the same theoretical line profiles used for Figure 1(b) but after modifying the data in Figure 1 (a) with the algorithm.

We find this algorithm to be equally effective for each of the three 1872 systems which we have built for use at CFHT, the Dominion Astrophysical Observatory, and the University of British Columbia. The value of $\alpha$ is similar for all three systems.

This research was supported by operating grants to
G.A.H.W. and B.C. from the Natural Sciences and Engineering Research Council of Canada.

\section{REFERENCES}

Campbell, B., Walker, G. A. H., and Yang, S. 1988, Ap. J., 331, 902. de Strobel, G. C., and Spite, M., eds. 1987, The Impact of Very High S/N Spectroscopy on Stellar Physics (Boston: Kluwer).

Walker, G. A. H. 1987, The Impact of Very High S/N Spectroscopy on Stellar Physics, ed. G. C. de Strobel and M. Spite (Boston: Kluwer), p. 39.

Walker, G. A. H., Johnson, R., and Yang, S. 1985, in Advances in Electronics and Electron Physics, ed. B. L. Morgan (London: Academic Press), 64A, p. 213. 